\title{
Implicaciones pedagógicas de la realidad aumentada para la mejora de la enseñanza de las ciencias en primaria
}

\section{Pedagogical implications of augmented reality for improving the teaching of the sciences in primary school}

\author{
Alicia González Pérez \\ Universidad de Extremadura \\ aliciagp@unex.es \\ Isabel Cerezo Cortijo \\ Universidad de Extremadura \\ icerezoc@alumnos.unex.es
}

Recibido: 21/09/2020

Aceptado: $21 / 11 / 2020$

Publicado: 01/12/2020

\section{RESUMEN}

La realidad aumentada (RA), con su capacidad para tender puentes entre lo real y lo virtual, abre nuevas oportunidades para construir atractivos entornos de aprendizaje en el actual contexto de crisis a consecuencia de la COVID-19. La RA se considera como uno de los medios pedagógicos para promover la calidad de la enseñanza en las próximas generaciones y es, por ello, que en este artículo se presenta una revisión sistemática de literatura de cómo se está implementando la RA en los centros de primaria para el aprendizaje de las ciencias naturales. Se han analizado en profundidad dieciocho artículos de revistas, todos ellos indexados en Web of Science y que han sido publicados en los últimos cinco años. El propósito de esta investigación ha sido conocer cómo influye el uso de la RA en la motivación, rendimiento académico y en la carga cognitiva del alumnado que estudia ciencias en primaria y con esta tecnología. También, se analizan los recursos de RA para la educación científica más citados en las investigaciones consultadas. Los resultados han mostrado que la RA mejora significativamente la motivación y rendimiento académico del alumnado durante su utilización. Con respecto a la carga cognitiva, se ha demostrado que la inclusión de la RA en la educación científica no conlleva una mayor carga mental para el alumnado. Algunos de los materiales basados en la RA que emergen del análisis de esta selección son las aplicaciones de exploración (libros aumentados, marcadores de RA y puntos de interés para activar la información digital), las herramientas de simulación y los juegos.

\section{PALABRAS CLAVE}

Realidad Aumentada; Educación Primaria; Enseñanza de la ciencia; Aprendizaje Móvil; Rendimiento académico.

\section{ABSTRACT}

Augmented reality $(A R)$, with its ability to bridge the real and the virtual, opens new opportunities to build attractive learning contexts in the current context of crisis as a result of COVID-19. AR is seen as one of the pedagogical means of promoting the quality of teaching in the coming generations and is therefore that this article presents a systematic literature review of how AR is being implemented in primary schools for science learning. The eighteen selected journal articles, indexed in Web of Science and covering the last five years, have been analysed in depth. The purpose has been to know how the use of AR influences the motivation, academic performance and cognitive burden of students in the study of science. It also analyses the AR resources for scientific education most cited in the research with which they have been worked. The results have shown that AR significantly improves students' motivation and academic performance during use. About cognitive burden, it has been shown that the inclusion of AR in scientific education does not carry a greater mental burden for students. Some of the AR materials emerging from the analysis of this selection are exploration applications (augmented books, AR markers, and points of interest to activate digital information), simulation tools, and games. 


\section{KEYWORDS}

Augmented Reality; Primary Education; Science teaching; Mobile Learning; Learning Performance.

\section{CITA RECOMENDADA}

González, A. \& Cerezo, I. (2020). Implicaciones pedagógicas de la realidad aumentada para la mejora de la enseñanza de las ciencias en primaria. Revista Interuniversitaria de Investigación en Tecnología Educativa, 9, 2-16. https://doi.org/10.6018/riite.444961

\section{Principales aportaciones del artículo y futuras líneas de investigación:}

- Identificar los elementos relevantes que están detrás de la implementación de la RA en las aulas de Educación Primaria para la mejora de la competencia en ciencias naturales.

- Analizar cómo influye el uso de la RA en el rendimiento académico y la motivación.

- Conocer si aumenta la carga cognitiva cuando se ponen en práctica actividades didácticas basadas en RA.

- Identificar recursos que utilizan la RA en ciencias.

\section{LA REALIDAD AUMENTADA EN LA EDUCACIÓN}

La crisis actual, como consecuencia de la COVID-19, ha puesto en jaque la educación. Estudiantes de todo el mundo se han visto obligados a quedarse en casa y hacer un seguimiento de las clases de forma aislada y virtual. En este contexto de emergencia, las aplicaciones móviles, en general, y la realidad aumentada (RA), en particular, pueden ofrecer experiencias inmersivas interesantes, que ayuden a los estudiantes a hacer más llevadero y placentero el proceso de aprendizaje. Esta nueva realidad, requiere repensar la educación e implementar metodologías de enseñanza y aprendizaje que tengan en cuenta no solo lo presencial sino también lo virtual, y que construyan contextos de aprendizaje más atractivos y de calidad. Por ello, los dispositivos móviles van a ser claves en la educación del futuro, ya que se han convertido en elementos indispensables para la formación, el seguimiento, la evaluación y el control de las actividades de aprendizaje. Según la UNESCO (2020) las escuelas tienen que ser ambiciosas e identificar la viabilidad y el impacto de futuros escenarios, que incluyan una mejor y más rápida conectividad. Esto ha de permitir que la escuela incorpore nuevas tecnologías como Internet de las cosas (IOT), RA y realidad virtual, robótica, competencias STEAM y otras que sean posibles.

Lo cierto es que la RA ha experimentado un enorme crecimiento en los últimos años (Alkhattabi, 2017). Fue definida por González-Pérez (2016) como una tecnología emergente que añade información digital en formato de imágenes, vídeos o audio al mundo real. Esto supone un cambio en la percepción que tienen los usuarios sobre una realidad, ya que se ve potenciada con la incorporación de información adicional y enriquecida, y que es generada por un dispositivo electrónico. Todo ello se realiza con dispositivos multisensoriales con un único soporte visual o pantalla (ordenador, tableta, teléfono inteligente o pizarra digital) y que cuentan con una cámara y un software o programa lector de reconocimiento de marcadores, activadores o elementos físicos a los que se asocia una información digital (Palomares, 2014).

Todas las posibilidades que proporcionan las aplicaciones de RA han permitido su irrupción en diferentes ámbitos de aplicación, convirtiéndose en una tecnología multidisciplinar. Roblizo y Cózar (2015) sostienen que, debido a su gran influencia, impregna todas las actividades humanas, laborales, formativas, académicas, de ocio y de consumo. "La RA se encuentra omnipresente en nuestra vida cotidiana" (Manuri \& Sanna, 2016, p. 18), siendo acogida con una gran aceptación entre los ciudadanos. Es indiscutible que "ningún fenómeno ha impactado de 
manera tan profunda y en ámbitos tan diversos" (De la Blanca et al., 2016, p.3). En el ámbito educativo, la RA destaca por su versatilidad y por las numerosas oportunidades educativas que ofrece. Ya en el EDUCAUSE Horizon Report (Alexander et al., 2019) se discutió sobre la RA y la creación de realidad mixta en términos de ofrecer un entorno de aprendizaje virtual-mixto y físicohíbrido a través del cual los estudiantes puedan navegar. Pero sin duda, el enfoque más interesante es el uso de esta tecnología para enriquecer y potenciar las experiencias educativas en contextos auténticos.

Las tendencias actuales en el ámbito educativo se dirigen hacia una paulatina sustitución de los ordenadores por tabletas y la consolidación del aprendizaje móvil como un modelo educativo que debe ser implementado en las escuelas (Area et al., 2014). Esto propicia el repensar nuevas formas de integrar la tecnología en el currículo y favorecer la innovación en los procesos de enseñanza y aprendizaje, permitiendo la incorporación de los recursos tecnológicos más actuales en las aulas a todos los niveles educativos. En este sentido, la RA se perfila como una de las tecnologías emergentes que deben ser consideradas en el contexto actual para la mejora de la educación.

Por tanto, la RA ha propiciado cambios sustanciales en los diseños curriculares y en la manera de concebir el proceso de enseñanza y aprendizaje (Fombona et al., 2017). Con su incorporación en las actividades didácticas, los estudiantes asumen un papel más protagonista en su propio aprendizaje, lo que conlleva un cambio del rol docente. El profesorado se convierte en el encargado de orientar el desarrollo de las aplicaciones, diseñar experiencias de aprendizaje, asesorar a los estudiantes en su interacción con la RA, evaluar y retroalimentar el aprendizaje. Esta transformación en la manera de adquirir conocimientos "facilita la sensación de vivir una experiencia innovadora, de alto impacto sensorial, que genera curiosidad y que resulta especialmente atractiva para generaciones que han crecido en entornos cada vez más dominados por la tecnología digital" (Escamilla et al., 2017, p.16).

La incorporación de elementos en diferentes formatos (Zhang et al., 2020) fomenta la comprensión de los contenidos. Además, la interacción con el objeto de aprendizaje propicia la inmersión de los estudiantes en contextos de información más tangibles (Escamilla et al., 2017) y el acceso a la información se lleva a cabo de una manera diferente, modificando las formas de aprender y mejorando el conocimiento de la realidad (Fombona et al., 2017; Hung et al., 2017).

Sin embargo, a pesar del cambio de tendencia en la educación, aún es necesario adoptar iniciativas innovadoras promovidas por los centros educativos (bottom - up) que asuman unos retos a medio o largo plazo que resuelvan las necesidades específicas del alumnado y que promuevan la formación técnica y el desarrollo de la competencia digital en los docentes. Es fundamental que los legisladores, investigadores y gestores de las organizaciones educativas adopten medidas encaminadas a reducir la brecha digital, social y educativa del alumnado y que sean acordes con la filosofía de la Agenda 2030 para el Desarrollo Sostenible (González-Pérez et al., 2020), solo así se podrá trabajar en el desarrollo de una educación de calidad y sostenible en el tiempo.

\section{MÉTODO}

En este apartado se hace referencia a los objetivos de la propuesta de investigación y a la justificación y selección del diseño metodológico llevado a cabo.

\subsection{Objetivos}

El objetivo principal de esta revisión sistemática de literatura es el siguiente:

- Identificar los elementos relevantes que están detrás de la implementación de la RA en las aulas de Educación Primaria para la mejora de la competencia en ciencias naturales.

A partir del objetivo general se han establecido tres objetivos específicos que se muestran a continuación:

- Analizar cómo influye el uso de la RA en el rendimiento académico y la motivación. 
- Conocer si aumenta la carga cognitiva de estudiantes de entre 6 y 12 años cuando se ponen en práctica actividades didácticas basadas en RA para el estudio de las ciencias.

- Identificar los recursos que utilicen la RA en ciencias durante la etapa de primaria, según las investigaciones consultadas.

\subsection{Metodología}

Considerar a los estudiantes del futuro como usuarios potenciales de la RA es una realidad $y$, por ello, se considera importante identificar los elementos clave que emergen del uso e implementación de esta tecnología para el aprendizaje. Se ha optado por hacer una revisión sistemática de literatura que, según Kitchenham (2004) es "un método que permite la evaluación e interpretación de todas las investigaciones a nuestro alcance que resulten relevantes para una pregunta de investigación, tema o evento de interés".

La investigación ha sido dividida en tres fases, siguiendo las pautas de Kitchenham (2004) para la realización de la revisión sistemática de literatura (RSL). Tal y como se muestra en la figura 1 , en primer lugar, se ha planificado la investigación, seleccionando las revistas, y definiendo los criterios de inclusión y exclusión para el estudio. En segundo lugar, se ha llevado a cabo la revisión, lo que ha incluido una selección de estudios y una extracción de datos con respecto al objeto de estudio. Y en un tercer lugar, se ha realizado el informe de la revisión, que incluye un análisis de los resultados y discusión de los resultados, tendencias y conclusiones.

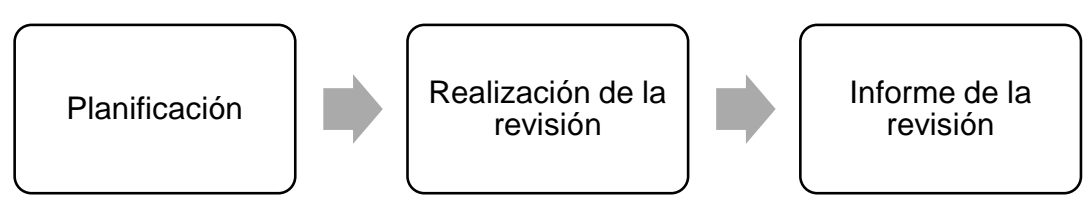

Figura 1. Procedimiento seguido para la revisión sistemática de literatura

\subsubsection{Criterios de inclusión y exclusión de la RSL}

Los criterios de inclusión/exclusión para el objeto de estudio han tenido en cuenta lo siguiente:

- Investigaciones publicadas en las revistas de tecnología educativa que estaban dentro del repositorio de Web of Science (WOS). (Tabla 1)

- Solamente se han incluido artículos de investigación.

- La tipología de los estudios de investigación analizados es: experimental, cuasi experimental, cualitativo, revisiones sistemáticas de literatura, y metaevaluaciones, fundamentalmente.

- Se han considerado investigaciones en lengua inglesa o española.

- El rango temporal de estudios seleccionados está entre 2015 y 2020.

- Únicamente se han considerado los artículos a texto completo en la Universidad de Extremadura o publicados en abierto.

Tras una primera lectura de los resúmenes y de los textos completos de los artículos seleccionados, se consideraron los artículos donde:

- El resumen y/o abstract respondía a los objetivos de la investigación.

- Eran investigaciones centradas en el uso de la RA en ciencias.

- Eran investigaciones centradas en el uso de la RA en primaria. 


\subsubsection{Protocolo de búsqueda}

La recopilación de la información de los estudios se ha realizado de manera exhaustiva y sistemática, seleccionando únicamente aquellas investigaciones que cumplían de manera estricta con los criterios mencionados anteriormente.

La selección de revistas que se muestra en la tabla 1 se ha llevado a cabo atendiendo a las revistas de tecnología educativa que obtuvieron un mayor factor de impacto en el año 2018.

Tabla 1. Selección de revistas internacionales y nacionales de tecnología educativa

\begin{tabular}{cc} 
Nombre de la revista & Factor de impacto (2018) \\
Computers \& Education & 5.627 \\
Comunicar & 3.338 \\
British Journal of Educational Technology & 2.588 \\
Journal of Computer Assisted Learning & 2.451 \\
Educational Technology Research and Development & 2.115 \\
Interactive Learning Environments & 1.929 \\
\hline
\end{tabular}

Las búsquedas en Web of Science arrojaron un total de 156 resultados, pero solamente 17 artículos pasaron la fase de lectura de los resúmenes y textos completos. En la tabla 2 se puede observar el protocolo de búsqueda seguido:

Tabla 2. Protocolo de búsqueda en la base de datos Web of Science

\begin{tabular}{|c|c|c|c|}
\hline $\begin{array}{l}\text { Palabras clave } \\
\text { /key words }\end{array}$ & Protocolo de búsqueda & $\begin{array}{l}\text { Resultados } \\
\text { parámetros de } \\
\text { inclusión y } \\
\text { exclusión }\end{array}$ & $\begin{array}{c}\text { Selección } \\
\text { tras leer el } \\
\text { resumen y } \\
\text { abstract }\end{array}$ \\
\hline $\begin{array}{l}\text { Augmented reality y } \\
\text { Primary Education }\end{array}$ & $\begin{array}{c}\text { Publicados } 2015 \text { - } 2020 \\
\text { Artículos de las revistas seleccionadas. } \\
\text { Inglés y español. }\end{array}$ & 55 & 11 \\
\hline Augmented reality y & Publicados 2015 - 2020 & & \\
\hline $\begin{array}{l}\text { Mobile learning y } \\
\text { Science }\end{array}$ & $\begin{array}{l}\text { Artículos de las revistas seleccionadas. } \\
\text { Inglés y español. }\end{array}$ & 68 & 3 \\
\hline Augmented reality y & Publicados 2015 - 2020 & & \\
\hline $\begin{array}{c}\text { Academic achievement } \\
\text { y Science }\end{array}$ & $\begin{array}{c}\text { Artículos de las revistas seleccionadas. } \\
\text { Inglés y español. }\end{array}$ & 7 & 1 \\
\hline $\begin{array}{l}\text { Augmented reality y } \\
\text { Electronic learning }\end{array}$ & $\begin{array}{c}\text { Publicados } 2015 \text { - } 2020 \\
\text { Artículos de las revistas seleccionadas. } \\
\text { Inglés y español. }\end{array}$ & 26 & 2 \\
\hline
\end{tabular}

A continuación, en la tabla 3 se muestran de manera sintética las ideas principales y características de los artículos seleccionados para la realización de la revisión sistemática de literatura. 
Tabla 3. Síntesis de los artículos de la RSL

\begin{tabular}{|c|c|c|c|c|c|}
\hline Artículo & Objetivo & $\begin{array}{c}\text { Tipo de } \\
\text { investigación }\end{array}$ & Muestra & Resultados & Conclusiones/ Prospectiva \\
\hline $\begin{array}{l}\text { (Arici et al., } \\
\text { 2019) }\end{array}$ & $\begin{array}{l}\text { Revelar las tendencias de investigación } \\
\text { con respecto al empleo de la RA en la } \\
\text { enseñanza de las ciencias. }\end{array}$ & $\begin{array}{l}\text { Análisis } \\
\text { bibliométrico }\end{array}$ & $\begin{array}{l}62 \text { artículos } \\
\text { indexados en } \\
\text { Web of } \\
\text { Science }\end{array}$ & $\begin{array}{l}\text { Las investigaciones entre los años } 2013 \text { y } 2018 \text { se } \\
\text { centran en el aprendizaje móvil y en los entornos } \\
\text { de aprendizaje electrónico. Además, las variables } \\
\text { aprendizaje, motivación y actitud son las más } \\
\text { analizadas. }\end{array}$ & $\begin{array}{c}\text { Son muy numerosos los estudios que evalúan las } \\
\text { variables de aprendizaje, motivación y actitud. Por } \\
\text { ello, se propone para futuras líneas de } \\
\text { investigación el análisis de cuestiones cognitivas, } \\
\text { de la interacción entre los estudiantes y de las } \\
\text { actividades cooperativas. }\end{array}$ \\
\hline $\begin{array}{l}\text { (Chang \& } \\
\text { Hwang, } \\
\text { 2018) }\end{array}$ & $\begin{array}{l}\text { Examinar la eficacia de la RA en un } \\
\text { aula invertida. }\end{array}$ & $\begin{array}{l}\text { Estudio } \\
\text { experimental }\end{array}$ & $\begin{array}{l}111 \\
\text { alumnos/as de } \\
10 \text { años }\end{array}$ & $\begin{array}{l}\text { La enseñanza mediante la inclusión de la RA en } \\
\text { un aula invertida fomentó el rendimiento } \\
\text { académico, la motivación, el pensamiento crítico y } \\
\text { la autoeficacia del grupo de los estudiantes. }\end{array}$ & $\begin{array}{l}\text { Los resultados obtenidos en el experimento } \\
\text { fueron muy favorables excepto en las pruebas de } \\
\text { conocimientos. Una posible causa es la } \\
\text { inexistencia de contenido práctico y que el tiempo } \\
\text { del experimento era demasiado corto como para } \\
\text { recordar los contenidos. }\end{array}$ \\
\hline $\begin{array}{c}\text { (Chen \& } \\
\text { Chen, 2018) }\end{array}$ & $\begin{array}{l}\text { Analizar si el empleo del sistema de } \\
\text { aprendizaje ubicuo basado en el } \\
\text { contexto mejora significativamente los } \\
\text { resultados de aprendizaje y la } \\
\text { motivación del alumnado. }\end{array}$ & $\begin{array}{l}\text { Estudio } \\
\text { experimental }\end{array}$ & $\begin{array}{l}86 \text { estudiantes } \\
\text { de } 12 \text { años }\end{array}$ & $\begin{array}{c}\text { Ha sido demostrado el efecto positivo del enfoque } \\
\text { de aprendizaje ubicuo basado en el contexto. La } \\
\text { confianza y motivación se han visto } \\
\text { incrementadas. }\end{array}$ & $\begin{array}{c}\text { Se espera para futuras investigaciones poder } \\
\text { abordar esta temática de una manera más amplia. }\end{array}$ \\
\hline $\begin{array}{c}\text { (Chen \& } \\
\text { Wang, 2018) }\end{array}$ & $\begin{array}{l}\text { Explorar la relación existente entre la } \\
\text { percepción del alumnado y su } \\
\text { presencia en los entornos de } \\
\text { aprendizaje con RA. }\end{array}$ & $\begin{array}{l}\text { Estudio } \\
\text { experimental }\end{array}$ & $\begin{array}{l}60 \text { alumnos/as } \\
\text { de } 11 \text { y } 12 \\
\text { años }\end{array}$ & $\begin{array}{c}\text { La presencia de los estudiantes en los entornos } \\
\text { de RA está directamente relacionada con los } \\
\text { resultados académicos. } \\
\text { Asimismo, una mejor percepción sobre la RA } \\
\text { conlleva una mayor presencia de los alumnos/as } \\
\text { en las actividades de aprendizaje. }\end{array}$ & $\begin{array}{l}\text { La RA tiene un gran potencial en el ámbito } \\
\text { educativo, pero aún son necesarias más } \\
\text { investigaciones que aborden esta tecnología } \\
\text { emergente desde una perspectiva psicológica. }\end{array}$ \\
\hline $\begin{array}{l}\text { (Fombona et } \\
\text { al., 2017) }\end{array}$ & $\begin{array}{l}\text { Delimitar las expectativas generadas } \\
\text { por la RA en la educación. }\end{array}$ & $\begin{array}{l}\text { Análisis } \\
\text { bibliométrico }\end{array}$ & $\begin{array}{l}67 \\
\text { investigaciones } \\
\text { indexadas en }\end{array}$ & $\begin{array}{l}\text { Destacan una serie de descriptores que se repiten } \\
\text { en la mayoría de las investigaciones. Son la } \\
\text { conceptualización del fenómeno, el desarrollo de } \\
\text { nuevas metodologías, la motivación generada, su }\end{array}$ & $\begin{array}{l}\text { Se están produciendo nuevos escenarios } \\
\text { educativos debido a los avances tecnológicos. } \\
\text { Esto supone que la RA se esté convirtiendo en un }\end{array}$ \\
\hline
\end{tabular}




\begin{tabular}{|c|c|c|c|c|c|}
\hline & & & $\begin{array}{l}\text { Web of } \\
\text { Science }\end{array}$ & $\begin{array}{l}\text { deslocalización espacial y las materias objeto de } \\
\text { implementación. }\end{array}$ & $\begin{array}{l}\text { elemento fundamental en las programaciones } \\
\text { didácticas. }\end{array}$ \\
\hline $\begin{array}{l}\text { (Hung et al. } \\
\text { 2017) }\end{array}$ & $\begin{array}{l}\text { Analizar la eficacia del libro gráfico de } \\
\text { RA frente a el libro de imágenes y las } \\
\text { interacciones físicas. }\end{array}$ & $\begin{array}{l}\text { Estudio } \\
\text { experimental }\end{array}$ & $\begin{array}{l}72 \text { personas } \\
\text { de } 12 \text { y } 13 \\
\text { años }\end{array}$ & $\begin{array}{l}\text { Los materiales de enseñanza que implementan la } \\
\text { RA facilitaron el aprendizaje de los diferentes tipos } \\
\text { de bacterias. Además, los participantes en el } \\
\text { experimento mostraron interés por el empleo de } \\
\text { materiales de RA. }\end{array}$ & $\begin{array}{c}\text { La RA no solo puede ayudar a aprender biología } \\
\text { (materia objeto de estudio en la investigación), } \\
\text { sino que también es posible utilizarla en otras } \\
\text { asignaturas. }\end{array}$ \\
\hline $\begin{array}{l}\text { (Hwang et } \\
\text { al., 2015) }\end{array}$ & $\begin{array}{c}\text { Conocer la eficacia del juego } \\
\text { competitivo de RA en comparación con } \\
\text { el enfoque tradicional del aprendizaje } \\
\text { móvil de RA. }\end{array}$ & $\begin{array}{l}\text { Estudio } \\
\text { experimental }\end{array}$ & $\begin{array}{l}57 \text { estudiantes } \\
\text { de } 11 \text { años }\end{array}$ & $\begin{array}{l}\text { El enfoque de juego competitivo de RA influye } \\
\text { positivamente en la actitud y el rendimiento } \\
\text { académico del alumnado cuando se planifican } \\
\text { actividades didácticas fuera de las aulas. }\end{array}$ & $\begin{array}{l}\text { Se concluye que la tecnología de códigos QR que } \\
\text { se adopta en la investigación tiene un bajo costo y } \\
\text { su implementación es fácil en los centros } \\
\text { educativos. } \\
\text { En prospectiva, sería conveniente tener en } \\
\text { consideración factores personales como los } \\
\text { estilos de aprendizaje o los niveles de } \\
\text { conocimientos en el enfoque propuesto. }\end{array}$ \\
\hline $\begin{array}{l}\text { (Hwang et } \\
\text { al., 2018) }\end{array}$ & $\begin{array}{l}\text { Comprobar el efecto del sistema de } \\
\text { aprendizaje basado en el } \\
\text { reconocimiento de imágenes en el logro } \\
\text { de aprendizaje, la motivación y la } \\
\text { identidad cultural local en Educación } \\
\text { Primaria. }\end{array}$ & $\begin{array}{l}\text { Estudio } \\
\text { experimental }\end{array}$ & $\begin{array}{l}105 \text { niños/as } \\
\text { de } 8 \text { años }\end{array}$ & $\begin{array}{l}\text { El sistema de aprendizaje móvil basado en el } \\
\text { reconocimiento de imágenes mejoró los logros de } \\
\text { aprendizaje, la motivación y la identidad cultural } \\
\text { local de los estudiantes. } \\
\text { También, se encontraron unos patrones de } \\
\text { aprendizaje más activo con respecto al grupo } \\
\text { control. }\end{array}$ & $\begin{array}{l}\text { Los resultados obtenidos se atribuyen a la } \\
\text { promoción activa en el aprendizaje, es decir, se } \\
\text { ofrece al alumnado consejos, una orientación } \\
\text { basada en preguntas y estímulos. } \\
\text { En un futuro, se plantea este enfoque de } \\
\text { aprendizaje contextual en otros cursos } \\
\text { académicos, incluyendo asignaturas como inglés. }\end{array}$ \\
\hline $\begin{array}{c}\text { (lbáñez \& } \\
\text { Delgado - } \\
\text { Kloos, 2018) }\end{array}$ & $\begin{array}{c}\text { Investigar las características de las } \\
\text { aplicaciones de RA en la enseñanza de } \\
\text { las ciencias, tecnología, ingeniería y } \\
\text { matemáticas. }\end{array}$ & $\begin{array}{l}\text { Revisión } \\
\text { sistemática de } \\
\quad \text { literatura }\end{array}$ & $\begin{array}{c}28 \\
\text { publicaciones } \\
\text { desde } 2010 \text { a } \\
2017\end{array}$ & $\begin{array}{c}\text { Un gran porcentaje de las aplicaciones de RA } \\
\text { para la enseñanza de las ciencias, tecnología, } \\
\text { ingeniería y matemáticas (STEM) tienen } \\
\text { actividades de exploración o simulación. } \\
\text { Por el contrario, muy pocos estudios ofrecieron a } \\
\text { los usuarios asistencia para la consecución de las } \\
\text { actividades. }\end{array}$ & $\begin{array}{l}\text { Es necesario diseñar características en las } \\
\text { aplicaciones para la adquisición de las } \\
\text { competencias básicas en las disciplinas STEM. } \\
\text { Además, se añade la importancia de incluir la } \\
\text { metacognición y el apoyo experimental en los } \\
\text { materiales didácticos de RA. }\end{array}$ \\
\hline
\end{tabular}




\begin{tabular}{|c|c|c|c|c|c|}
\hline $\begin{array}{l}\text { (Kumar et } \\
\text { al., 2018) }\end{array}$ & $\begin{array}{c}\text { Conocer la efectividad del uso de la } \\
\text { evaluación formativa basada en la RA } \\
\text { para la mejora del aprendizaje y la } \\
\text { motivación. }\end{array}$ & $\begin{array}{c}\text { Estudio } \\
\text { comparativo }\end{array}$ & $\begin{array}{l}70 \text { alumnos/as } \\
\text { de Educación } \\
\text { Primaria }\end{array}$ & $\begin{array}{l}\text { La utilización de la RA en la evaluación formativa } \\
\text { mejoró de manera significativa el aprendizaje y la } \\
\text { motivación del alumnado en comparación con el } \\
\text { método de enseñanza tradicional. }\end{array}$ & $\begin{array}{c}\text { Es recomendable la evaluación formativa basada } \\
\text { en la RA para la enseñanza de la educación } \\
\text { ambiental y las habilidades intelectuales simples } \\
\text { del alumnado de Educación Primaria. }\end{array}$ \\
\hline $\begin{array}{l}\text { (Kyza \& } \\
\text { Georgiou, } \\
\text { 2018) }\end{array}$ & $\begin{array}{l}\text { Evaluar la plataforma de RA } \\
\text { TraceReaders y las aplicaciones } \\
\text { Mystery at the Lake y Young } \\
\text { Archaeologists. }\end{array}$ & $\begin{array}{l}\text { Estudio de } \\
\quad \text { caso }\end{array}$ & $\begin{array}{l}29 \text { estudiantes } \\
\text { de } 7 \text { años y } 11 \\
\text { alumnos/as de } \\
15 \text { años }\end{array}$ & $\begin{array}{l}\text { Las aplicaciones Mystery at the Lake y Young } \\
\text { Archaeologists apoyan el aprendizaje in situ e } \\
\text { involucran al alumnado en actividades de } \\
\text { investigación y de resolución de problemas. } \\
\text { Se ha observado que la planificación de las } \\
\text { actividades no es suficiente para la comprensión } \\
\text { de los contenidos. En cambio, las discusiones } \\
\text { reflexivas y de resolución de problemas } \\
\text { demostraron ser más efectivas. }\end{array}$ & $\begin{array}{l}\text { Los resultados han puesto de manifiesto la } \\
\text { necesidad de investigaciones que aborden la } \\
\text { introducción de estas plataformas en las } \\
\text { asignaturas de ciencias con mayor profundidad. }\end{array}$ \\
\hline $\begin{array}{l}\text { (Lai et al., } \\
\text { 2019) }\end{array}$ & $\begin{array}{l}\text { Investigar sobre los efectos del libro } \\
\text { multimedia de RA en los logros y } \\
\text { motivaciones de aprendizaje de los } \\
\text { estudiantes de Educación Primaria. }\end{array}$ & $\begin{array}{c}\text { Estudio } \\
\text { experimental }\end{array}$ & $\begin{array}{l}46 \text { alumnos/as } \\
\text { de } 10 \text { y } 11 \\
\text { años }\end{array}$ & $\begin{array}{l}\text { El alumnado que aprendió los contenidos de } \\
\text { ciencias con el libro multimedia de RA obtuvo } \\
\text { unas puntuaciones mayores en el logro } \\
\text { académico que en la motivación. }\end{array}$ & $\begin{array}{l}\text { La tecnología de RA en la lectura científica tiene } \\
\text { múltiples ventajas. } \\
\text { El experimento ha sido llevado a cabo a pequeña } \\
\text { escala, por lo que sería conveniente realizarlo } \\
\text { gran escala o mediante una encuesta cuantitativa. }\end{array}$ \\
\hline $\begin{array}{l}\text { (Laine et al., } \\
\text { 2016) }\end{array}$ & $\begin{array}{c}\text { Evaluar la plataforma Science Spots } A R \\
\text { utilizando como prueba el juego } \\
\text { Leometry. }\end{array}$ & $\begin{array}{l}\text { Mixta: } \\
\text { Cuestionarios } \\
\text { y entrevistas }\end{array}$ & $\begin{array}{c}61 \text { niños/as de } \\
12 \text { años }\end{array}$ & $\begin{array}{c}\text { Science Spots } A R \text { presenta pequeñas } \\
\text { deficiencias, pero es factible y tiene potencial para } \\
\text { la construcción de juegos de aprendizaje sobre } \\
\text { ciencias. } \\
\text { Permite la enseñanza en cualquier entorno a } \\
\text { través de experiencias de aprendizaje } \\
\text { contextualizadas. }\end{array}$ & $\begin{array}{c}\text { La prospectiva de la investigación se dirige hacia } \\
\text { la evaluación de la dimensión pedagógica, } \\
\text { contextual y motivacional de Science } \\
\text { Spots } A R .\end{array}$ \\
\hline $\begin{array}{l}\text { (Suárez et } \\
\text { al., 2017) }\end{array}$ & $\begin{array}{c}\text { Examinar cómo la enseñanza por } \\
\text { indagación apoya y limita el aprendizaje } \\
\text { de los estudiantes. }\end{array}$ & $\begin{array}{l}\text { Revisión } \\
\text { sistemática de } \\
\text { literatura }\end{array}$ & $\begin{array}{c}62 \\
\text { investigaciones }\end{array}$ & $\begin{array}{l}\text { Las actividades móviles basadas en el } \\
\text { aprendizaje basado en la indagación se clasifican } \\
\text { en instrucción directa, acceso al contenido, } \\
\text { recopilación de datos, comunicación entre pares y } \\
\text { apoyo contextual. }\end{array}$ & $\begin{array}{c}\text { La mayoría de los documentos encontrados en la } \\
\text { revisión sistemática de literatura son estudios de } \\
\text { caso debido a que existen muy pocos estudios } \\
\text { experimentales. } \\
\text { Esto ha supuesto la dificultad de llegar a una } \\
\text { conclusión acerca de la temática objeto de } \\
\text { estudio. }\end{array}$ \\
\hline
\end{tabular}




\begin{tabular}{|c|c|c|c|c|c|}
\hline $\begin{array}{c}\text { (Weng et al., } \\
\text { 2019) }\end{array}$ & $\begin{array}{l}\text { Explorar si la inclusión de la realidad } \\
\text { mixta utilizada en un libro de ciencias } \\
\text { puede mejorar los resultados } \\
\text { académicos. }\end{array}$ & $\begin{array}{c}\text { Estudio } \\
\text { experimental }\end{array}$ & $\begin{array}{l}80 \text { personas } \\
\text { de entre } 9 \text { y } 11 \\
\quad \text { años }\end{array}$ & $\begin{array}{c}\text { El empleo de la realidad mixta como apoyo al libro } \\
\text { impreso tradicional mejora el aprendizaje, } \\
\text { especialmente en aquellos estudiantes con una } \\
\text { baja habilidad espacial. }\end{array}$ & $\begin{array}{l}\text { Hay muchas investigaciones sobre cómo la } \\
\text { realidad mixta aumenta la motivación y } \\
\text { participación. Sin embargo, son muy pocas las } \\
\text { que indagan en su impacto en el logro del } \\
\text { alumnado. }\end{array}$ \\
\hline $\begin{array}{c}\text { (Wu et al., } \\
\text { 2018) }\end{array}$ & $\begin{array}{l}\text { Comparar el aprendizaje con RA } \\
\text { basado en herramientas mentales con } \\
\text { el sistema tradicional teniendo en } \\
\text { cuenta la mejora del aprendizaje en } \\
\text { ciencias. }\end{array}$ & $\begin{array}{c}\text { Estudio } \\
\text { experimental }\end{array}$ & $\begin{array}{l}50 \text { niños/as de } \\
9 \text { y } 10 \text { años }\end{array}$ & $\begin{array}{c}\text { Se obtuvo una mejora en los logros de } \\
\text { aprendizaje y no se encontraron diferencias en } \\
\text { términos de carga cognitiva entre ambos } \\
\text { sistemas. }\end{array}$ & $\begin{array}{c}\text { La incorporación de las herramientas mentales y } \\
\text { de la RA en la educación ayuda a la construcción } \\
\text { de conocimientos y a la mejora de los logros de } \\
\text { aprendizaje. }\end{array}$ \\
\hline $\begin{array}{l}\text { (Zhang et } \\
\text { al., 2020) }\end{array}$ & $\begin{array}{l}\text { Diseñar una hoja de trabajo de RA con } \\
\text { un aprendizaje sobre las plantas } \\
\text { basado en tres etapas (exploración, } \\
\text { introducción conceptual y la aplicación } \\
\text { del concepto). }\end{array}$ & $\begin{array}{l}\text { Estudio cuasi } \\
\text { experimental }\end{array}$ & $\begin{array}{l}89 \text { niños/as de } \\
8 \text { años }\end{array}$ & $\begin{array}{l}\text { Los resultados de aprendizaje obtenidos con la } \\
\text { utilización de las hojas de trabajo basadas en la } \\
\text { RA son significativamente mejores y propician la } \\
\text { interacción de los estudiantes con las plantas. }\end{array}$ & $\begin{array}{c}\text { Se concluye que las hojas de trabajo basadas en } \\
\text { la RA permiten al alumnado prestar más atención } \\
\text { a los objetos físicos y proporcionan contenido } \\
\text { multimedia más rico que las hojas de trabajo en } \\
\text { papel. }\end{array}$ \\
\hline
\end{tabular}




\section{RESULTADOS}

En esta sección se describen y se presentan los resultados obtenidos de la revisión sistemática de literatura, atendiendo a los objetivos planteados en la investigación y considerando las investigaciones seleccionadas en la sección metodológica.

\subsection{Influencia del uso de la realidad aumentada en el rendimiento académico y la motivación hacia las ciencias entre el alumnado de Educación Primaria}

Los artículos seleccionados en esta revisión sistemática de literatura han puesto de manifiesto la pertinencia de analizar la implementación de la RA en la asignatura de ciencias en primaria, para ver cómo afecta el uso de esta tecnología en el rendimiento académico y a la motivación del alumnado (Arici et al.,2019; Chen \& Chen, 2018; Suárez et al., 2017; Zhang et al., 2020).

En términos de rendimiento académico, son numerosas las investigaciones que abordan esta variable y muchas de ellas inciden en los efectos positivos que produce el uso de la RA en el ámbito educativo (Arici et al., 2019; Chang \& Hwang, 2018). Aunque tradicionalmente, la medición del rendimiento académico ha sido llevada a cabo con otro tipo de recursos didácticos o enfoques metodológicos, es importante destacar los hallazgos de la investigación realizada por Hwang et al. (2018). Los resultados experimentales mostraron que el aprendizaje móvil basado en la RA y apoyado en elementos de la cultura local mejoró el aprendizaje de los estudiantes, su motivación y se activaron mecanismos del aprendizaje activo. En el estudio experimental llevado a cabo por Hwang et al. (2015) para examinar la eficacia del juego competitivo a través de RA en la etapa de Educación Primaria, se arrojaron diferencias muy significativas entre el grupo control y experimental. En este último, se dieron efectos positivos en la adquisición de logros y actitudes con respecto a la eficacia del juego competitivo con RA.

La participación activa en las actividades de aprendizaje también juega un papel crucial en los resultados académicos. La enseñanza basada en RA es un mecanismo activo de promoción del aprendizaje que mejora notablemente los logros del alumnado de Educación Primaria (Hwang et al., 2018).

Por el contrario, en otro experimento en el que se analizó la eficacia de la RA en un aula invertida, no se encontraron evidencias significativas de un aumento de los beneficios, en cuanto a los logros de aprendizaje obtenidos, en comparación con el enfoque convencional (Chang \& Hwang, 2018).

Habitualmente, la inclusión de la RA en los centros educativos va asociada a una presencia física, social y temporal del alumnado en las actividades didácticas (Chen \& Wang, 2018). La relación entre las variables presencia y rendimiento de aprendizaje es significativa. En palabras de Chen y Wang (2018), los entornos de aprendizaje mediados se correlacionan con un bajo rendimiento de aprendizaje por parte de los estudiantes. A pesar de ello, una presencia del alumnado en las actividades basadas en RA no garantiza un alto rendimiento académico.

Otro posible motivo de la eficacia de la RA es la retroalimentación con refuerzo positivo que ofrecen la mayoría de los sistemas de RA. Cuando se desarrollan actividades en entornos de aprendizaje interactivos y activos se ve incrementada la motivación de los estudiantes hacia los contenidos de ciencias que imparte el docente (Kumar et al., 2018). A ello se suma, la novedad de esta tecnología emergente, que puede funcionar como un elemento motivador (Fombona et al., 2017; Laine et al., 2016).

Según algunas investigaciones, el alumnado con discapacidad y/o trastornos de aprendizaje, como, por ejemplo, el trastorno por déficit de atención e hiperactividad se ve mucho más beneficiado por esta tecnología emergente, mostrando un aumento significativo en la motivación y el nivel de conocimiento (Ibáñez \& Delgado-Kloos, 2018). Asimismo, el alumnado con bajo y alto rendimiento ven mejorada su cognición y, por consiguiente, sus calificaciones. 
Sin embargo, aún es necesaria una mayor investigación de la utilidad pedagógica de la RA desde una perspectiva psicológica y pedagógica (Chen \& Wang, 2018). Es de suma importancia considerar las características específicas del alumnado (Ibáñez \& Delgado-Kloos, 2018) como la edad, la duración de la intervención y el entorno de aprendizaje físico como variables a estudiar, y para evaluar los resultados de aprendizaje. Por ello, se requiere de una mayor investigación con respecto a los efectos pedagógicos y psicológicos que tiene en el alumnado el uso e implementación de la RA en ambientes tecnologizados.

\subsection{Carga cognitiva de estudiantes de entre 6 y 12 años cuando se ponen en práctica actividades didácticas basadas en el empleo de la realidad aumentada para la enseñanza de las ciencias naturales}

Esta sección aborda la segunda pregunta de investigación, y que va en la línea de conocer si los estudiantes pueden sufrir sobrecarga cognitiva cuando aprenden en un entorno de RA. En las investigaciones revisadas existe unanimidad en la importancia de analizar la carga cognitiva del alumnado cuando aprenden en un entorno de RA, ya que necesita enfrentarse tanto a información del mundo real como digital. Este enfoque de aprendizaje lleva implícito que tiene que haber un diseño adecuado de los materiales y de las tareas de aprendizaje en RA. Según Ke y Hsu (2015) para ayudar a los estudiantes a interpretar la rica información obtenida de los contextos de aprendizaje basados en RA, es importante tener una herramienta de apoyo.

La carga cognitiva se considera una construcción multidimensional del sistema cognitivo del alumnado, que expresa la carga experimentada al realizar una tarea en particular, y consta de tres aspectos a considerar: la carga mental, el esfuerzo mental y el rendimiento.

En el estudio de Wu et al. (2018) se llegó a la conclusión que los estudiantes que aprendieron con una "herramienta mental" basada en un enfoque de aprendizaje en RA superaron al grupo de estudiantes que aprendieron con un enfoque de aprendizaje en RA convencional, en términos de logros de aprendizaje. Además, pasaron un cuestionario al alumnado de 9 y 10 años para analizar la carga cognitiva en dos dimensiones: carga y esfuerzos mentales. Wu et al. (2018) determinaron que no existían diferencias significativas entre las calificaciones obtenidas en cuanto a la carga mental del grupo control y experimental, cuando se implementaba un sistema de aprendizaje de RA basado en herramientas mentales. En lo que respecta a la dimensión de esfuerzo mental, tampoco se obtuvieron diferencias significativas entre ambos grupos. Cabe destacar que todos los valores de los cuestionarios se encontraban por debajo de la media, lo que indica que los dos grupos percibieron una baja carga cognitiva durante la realización de la actividad didáctica.

Lai et al. (2019) diseñaron un sistema de aprendizaje basado RA, donde integraron diversos materiales multimedia para explorar los efectos de este enfoque en la eficacia de los aprendizajes. Los resultados experimentales mostraron que este enfoque promovió significativamente los aprendizajes en los estudiantes y la motivación. Según los datos, los estudiantes que aprenden con RA reducen la carga cognitiva en comparación con el alumnado que aprende con el método convencional multimedia sobre ciencia.

Estos resultados coinciden con los obtenidos en una investigación llevada a cabo por Chang y Hwang (2018), en la que se analizó la carga cognitiva del alumnado que aprende contenidos de ciencias mediante la RA en un aula invertida. A partir de los resultados experimentales, se concluyó que el enfoque de aula invertida basada en RA no solo benefició a los estudiantes en términos de desempeño, sino que también mejoró su motivación por el aprendizaje, el pensamiento crítico, la carga cognitiva y la autoeficacia grupal.

\subsection{Recursos con realidad aumentada para la educación científica en Educación Primaria}

En este apartado se presentan algunos de los materiales de RA diseñados para la educación científica del alumnado de primaria (6 y 12 años), y que han sido seleccionados a partir de la muestra de artículos que han configurado esta investigación. 
En la revisión sistemática de literatura realizada por Ibáñez y Delgado-Kloos (2018) emergieron tres grandes grupos de aplicaciones educativas con RA para la enseñanza de las ciencias. Según estos autores podrían caracterizarse por ser aplicaciones de exploración (libros aumentados, marcadores de RA y puntos de interés para activar la información digital), herramientas de simulación y juegos.

Los libros aumentados, enmarcados dentro de las aplicaciones de exploración, son aplicaciones de RA diseñadas en forma de libro (Arici et al., 2019). Este recurso posibilita la elección del punto de vista más apropiado para presentar modelos virtuales en 3D que aparecen reflejados en las páginas del libro (Ibáñez \& Delgado-Kloos, 2018).

Hung et al. (2017) analizaron la eficacia del uso de un libro gráfico de RA frente a un libro de imágenes, y su interacción con el usuario. A través de estos materiales se mostraban las características de seis tipos de bacterias mediante gráficos en 2D, objetos físicos en $3 \mathrm{D}$ y objetos virtuales en $3 \mathrm{D}$ a estudiantes de entre 12 y 13 años. El libro gráfico de RA estaba formado por seis páginas y cada una de ellas contenía un marcador de seguimiento, el nombre de una bacteria y una descripción de esta. Los resultados demostraron que había una correlación positiva entre el tiempo de uso y el tipo de material, ya que afectó a las calificaciones y al número de errores cometidos. Por tanto, la RA en este caso produjo beneficios educativos entre los estudiantes, y éstos mostraron una gran satisfacción e interés por su uso en la asignatura de biología.

Weng et al. (2019) entrevistaron a un grupo de estudiantes tras una sesión didáctica con el libro de realidad mixta y los resultados fueron muy positivos. Se obtuvieron respuestas como: "Puedo leer la información en este libro y puedo hacer que cobre vida. Aprender ciencias me hace sentir bien". Otro estudiante afirmó que "no necesitaba imaginar el proceso del eclipse solar y lunar, ya que, con el 3D era muy fácil reproducirlo en su mente".

Los marcadores de RA son claves visuales, que pueden ser imágenes, logos, objetos 2D que activan información digital y que pueden usar la ubicación con la ayuda del GPS (Arici et al., 2019). En esta última categoría se puede citar la plataforma TraceReaders $R A$, que permite la creación de aplicaciones de RA basadas en la ubicación para el aprendizaje en entornos al aire libre (Kyza \& Georgiou, 2018).

Las herramientas de simulación únicamente han sido citadas en el artículo de lbáñez y Delgado-Kloos (2018) y no se han localizado evidencias de su efectividad. También se pueden encontrar juegos desarrollados en SSAR, que permiten concretar y simular conceptos científicos que en muchas ocasiones resultan abstractos a través de la combinación de objetos reales y virtuales de una manera amena y divertida (Laine et al., 2016).

\section{DISCUSIÓN Y CONCLUSIONES}

En esta investigación se pone en valor la importancia de la RA como una tecnología emergente, que abre nuevas oportunidades para construir contextos de aprendizaje más atractivos y constructivos. En este momento de transformación digital de la enseñanza, la RA se posiciona como un medio pedagógico en alza para la próxima generación. Por ello, se hace necesario ir más allá de las cuestiones puramente técnicas que tienen que ver con la implementación de esta tecnología. Y desde la comunidad científica se demandan estudios rigurosos que presten atención al impacto de esta tecnología en la práctica del aula, para así tomar decisiones clave sobre cuándo y cómo, la RA es pertinente y eficaz para mejorar los procesos de enseñanza-aprendizaje.

Los artículos seleccionados para la realización de la revisión sistemática de literatura $(n=17)$ han puesto de manifiesto que poner en práctica un enfoque de aprendizaje basado en la RA tiene efectos positivos en el rendimiento académico (Arici et al., 2019; Chang \& Hwang, 2018), en la motivación del estudiante, en la activación de mecanismos de aprendizaje activo (Hwang et al., 2018), y en la mejora de la carga cognitiva (Chang \& Hwang, 2018; Lai et al., 2019; Wu et al., 2018). Sin embargo, López-Faican y Jaen (2020) advierten que un mal diseño e integración de los contenidos elaborados con RA en contextos de aprendizaje científico, puede suponer un incremento considerable de la carga cognitiva para el alumnado. Por lo tanto, es de vital 
importancia diseñar cuidadosamente la presentación de los materiales y las tareas en sistemas de RA para reducir la carga cognitiva de los estudiantes.

Así pues, el uso de la RA en la educación tiene enormes beneficios en la enseñanza cuando se intenta aprovechar todo el potencial interactivo, colaborativo e innovador en pro de una nueva cultura de aprendizaje. Según se observa en la literatura revisada, involucrar a los estudiantes en otras formas de hacer supone un incremento de la motivación (Weng et al., 2019), favorece nuevas formas de interactuar en entornos inmersivos y extiende la práctica tradicional a otras dimensiones virtuales.

Estrechamente relacionadas con la carga cognitiva se encuentran las variables rendimiento académico y motivación. Los efectos positivos de la tecnología de RA en el rendimiento académico y motivación del alumnado de Educación Primaria son evidentes (Chiang et al., 2014). Pero aún se precisa una mayor investigación desde una perspectiva psicológica por considerarse limitada. En este sentido, es importante abordar nuevas líneas de investigación que consideren las características personales del alumnado, como pueden ser la edad y los estilos de aprendizaje. Arici et al. (2019) recomiendan para futuras líneas de investigación abordar el análisis de los problemas cognitivos que afronta el alumnado, la interacción y las actividades colaborativas a través de esta tecnología.

Finalmente, cabe destacar que los beneficios de la RA para la enseñanza científica no radican únicamente en la inclusión de esta tecnología en la educación, sino que es fundamental la elección de recursos didácticos adecuados y su correcta utilización. La revisión sistemática de literatura ha arrojado tres recursos con RA que destacan por los resultados positivos en el aprendizaje del alumnado. Son los libros aumentados, los marcadores de RA y los juegos de RA.

Probablemente, si en nuestro estudio no se hubiesen puesto limitaciones de acceso a otras bases de datos, así como, considerar solo recursos a texto completo y abierto, quizás hubiésemos obtenido una muestra más amplia de estudios relacionados con el objeto de estudio. En la revisión sistemática de literatura llevada a cabo se ha visto que son pocos los estudios que abordan la RA, desde la perspectiva de la enseñanza científica. Por tanto, habría que explorar nuevas búsquedas al respecto, considerando otros términos de búsqueda y considerando otras bases de datos especializadas en el campo de la educación como: SCOPUS, EBSCO, DIALNET, ERIC y SCILIT.

\section{REFERENCIAS BIBLIOGRÁFICAS}

Alexander, B., Ashford-Rowe, K., Barajas-Murphy, N., Dobbin, G., Knott, J., McCormack, M., \& Weber, N. (2019). EDUCAUSE Horizon Report: 2019 Higher Education Edition. EDUCAUSE.

Alkhattabi, M. (2017). Augmented reality as e-learning tool in primary schools' education: Barriers to teachers' adoption. International Journal of Emerging Technologies in Learning, 12(2), $91-$ 100. https://doi.org/10.3991/ijet.v12i02.6158

Area, M., Alonso, C., Correa, J.M., Del Moral, M.E., de Pablos, J., Paredes, J., Peirats, J., Sanabria, A.L., San Martín, A., \& Valverde, J. (2014). Las políticas educativas TIC en España después del Programa Escuela 2.0: Las tendencias que emergen. Revista Latinoamericana de Tecnología Educativa, 13(2), 11-33. https://doi.org/10.17398/1695-288X.13.2.11

Arici, F., Yildirim, P., Caliklar, Ş., \& Yilmaz, R.M. (2019). Research trends in the use of augmented reality in science education: Content and bibliometric mapping analysis. Computers and Education, 142, 1-23. https://doi.org/10.1016/i.compedu.2019.103647

Chang, S.C., \& Hwang, G.J. (2018). Impacts of an augmented reality-based flipped learning guiding approach on students' scientific project performance and perceptions. Computers and Education, 125, 226-239. https://doi.org/10.1016/j.compedu.2018.06.007

Chen, C.C., \& Chen, C.Y. (2018). Exploring the effect of learning styles on learning achievement in a u-Museum. Interactive Learning Environments, 26(5), 664-681. https://doi.org/10.1080/10494820.2017.1385488 
Chen, Y.H., \& Wang, C.H. (2018). Learner presence, perception, and learning achievements in augmented-reality-mediated learning environments. Interactive Learning Environments, 26(5), 695-708. https://doi.org/10.1080/10494820.2017.1399148

Chiang, T.H.C., Yang, S.J.H., \& Hwang, G.J. (2014). An augmented reality-based mobile learning system to improve students' learning achievements and motivations in natural science inquiry activities. Educational Technology \& Society, 17(4), 352-365. https://bit.ly/3flyp8I

De la Blanca, S., Chicharro, J., \& Moreno, E. (2016). Realidad aumentada y proyectos de trabajo. Un maridaje con proyección. [Conference]. Universidad de Sevilla, Sevilla, España. https://bit.ly/35S4dit

Escamilla, J., Venegas, E., Fernández, K., Fuerte, K., Román, R., Abrego, G., Murillo, A., \& Guijosa, C. (2017). Realidad Aumentada y Virtual. EduTrends. https://bit.ly/2UMtrbB

Fombona, J., Pascual-Sevillano, M.A., \& González-Videgaray, M. (2017). M-learning y realidad aumentada: Revisión de literatura científica en el repositorio WoS. Comunicar, 25(52), 63-72. https://doi.org/10.3916/C52-2017-06

González-Pérez, A. (2016). Innovación tecnológica en la creación de recursos educativos. In J.A. Camisión, S. Soriano, L. Chano, J. Díaz (Eds.), Experiencias de Innovación Docente En Los Estudios Jurídicos: Una Visión Práctica (pp. 9-14). Servicio Publicaciones UEX. https://bit.ly/31Ta50z

González-Pérez, A. (2018). Social Networks as Tools to Enrich Learning Environment in Higher Education. Bordón. Revista de Pedagogía, 70(4), 55-71. https://doi.org/10.13042/Bordon.2018.60579

González-Pérez, A., Sosa, M.J., \& Martínez, J.M. (2020). Políticas Educativas para la Integración de las TIC en el Sistema Educativo. ¿Vamos hacia Políticas Inclusiva, Equitativas y de Calidad? Revista Viceversa, 110, 64-69. https://bit.ly/3kQVHoi

Hung, Y.H., Chen, C.H., \& Huang, S.W. (2017). Applying augmented reality to enhance learning: A study of different teaching materials. Journal of Computer Assisted Learning, 33(3), 252266. https://doi.org/10.1111/ical.12173

Hwang, G.J., Chang, S.C., Chen, P.Y., \& Chen, X.Y. (2018). Effects of integrating an active learning-promoting mechanism into location-based real-world learning environments on students' learning performances and behaviors. Educational Technology Research and Development, 66(2), 451-474. https://doi.org/10.1007/s11423-017-9567-5

Hwang, G.J., Wu, P.H., Chen, C.C., \& Tu, N.T. (2015). Effects of an augmented reality-based educational game on students' learning achievements and attitudes in real-world $\begin{array}{llll}\text { observations. Interactive Learning Environments, 24(8), } & \text { 1-12. }\end{array}$ https://doi.org/10.1080/10494820.2015.105774 7

Ibáñez, M.B., \& Delgado-Kloos, C. (2018). Augmented reality for STEM learning: A systematic review. Computers \& Education, 123, 109-123. https://doi.org/10.1016/j.compedu.2018.05.002462

Ke, F., \& Hsu, Y. C. (2015). Mobile augmented-reality artifact creation as a component of mobile computer-supported collaborative learning. The Internet and Higher Education, 26, 33-41. https://doi.org/10.1016/j.iheduc.2015.04.003

Kitchenham, B. (2004). Procedures for Performing Systematic Reviews. UK and Autralia: Joint Technical Report.

Kumar, K., Liou, W.K., Spector, J.M., \& Chang, C.Y. (2018). To use augmented reality or not in formative assessment: A comparative study. Interactive Learning Environments, 27(5), 830840. https://doi.org/10.1080/10494820.2018.1489857

Kyza, E.A., \& Georgiou, Y. (2018). Scaffolding augmented reality inquiry learning: The design and investigation of the TraceReaders location-based, augmented reality platform. Interactive Learning Environments, 27(2), 211-225. https://doi.org/10.1080/10494820.2018.1458039 
Lai, A.F., Chen, C.H., \& Lee, G.Y. (2019). An augmented reality-based learning approach to enhancing students' science reading performances from the perspective of the cognitive load theory. British Journal of Educational Technology, 50(1), 232-247. https://doi.org/10.1111/bjet.12716

Laine, T.H., Nygren, E., Dirin, A., \& Suk, H.J. (2016). Science Spots AR: A platform for science learning games with augmented reality. Educational Technology Research and Development, 64(3), 507-531. https://doi.org/10.1007/s11423-015-9419-0

López-Faican, L., \& Jaen, J. (2020). EmoFindAR: Evaluation of a mobile multiplayer augmented reality game for primary school children. Computers and Education, 149(1), 1-20. https://doi.org/10.1016/i.compedu.2020.103814

Manuri, F., \& Sanna, A. (2016). A Survey on Applications of Augmented Reality. Advances in Computer Science: An International Journal, 5(19), 18-27. https://bit.ly/2UK5wta

Palomares, M.C. (2014). La Realidad Aumentada en la comunicación literaria: El caso de los libros interactivos. Ensayos: Revista de La Facultad de Educación de Albacete, 29(2), 79-94. https://doi.org/10.18239/ensayos.v29i2.385

Roblizo, M.J., \& Cózar, R. (2015). Usos y competencias en TIC en los futuros maestros de educación infantil y primaria: Hacia una alfabetización tecnológica real para docentes. PíxelBit, Revista de Medios y Educación, 47, 23-39. https://bit.ly/35QQxEn

Suárez, A., Specht, M., Prinsen, F., Kalz, M., \& Ternier, S. (2017). A review of the types of mobile activities in mobile inquiry-based learning. Computers \& Education, 118(5), 38-55. https://doi.org/10.1016/i.compedu.2017.11.004

UNESCO (2020). The digital Transformation of Education: Connecting Schools, Empowering Learners. Switzerland: Broadband Commission for Sustainable Development.

Weng, C., Rathinasabapathi, A., Weng, A., \& Zagita, C. (2019). Mixed Reality in Science Education as a Learning Support: A Revitalized Science Book. Journal of Educational Computing Research, 57(3), 777-807. https://doi.org/10.1177/0735633118757017

Wu, P.H., Hwang, G.J., Yang, M.L., \& Chen, C.H. (2018). Impacts of integrating the repertory grid into an augmented reality-based learning design on students' learning achievements, cognitive load and degree of satisfaction. Interactive Learning Environments, 26(2), 221-234. https://doi.org/10.1080/10494820.2017.1294608

Zhang, J., Huang, Y.T., Liu, T.C., Sung, Y.T., \& Chang, K.E. (2020). Augmented reality worksheets in field trip learning. Interactive Learning Environments, 5(20),1-18. https://doi.org/10.1080/10494820.2020.1758728

\section{INFORMACIÓN SOBRE LAS AUTORAS}

\section{Alicia González Pérez \\ Universidad de Extremadura}

Profesora Contratada Doctora de la Universidad de Extremadura. Doctora Europea en Ciencias de la Educación (2011) y Premio Extraordinario de Doctorado (2014) por la Universidad de Sevilla. Es autora de numerosos artículos, capítulos de libro y libros, publicados en revistas y editoriales de reconocido prestigio, sobre políticas educativas TIC, innovación educativa, tecnología educativa, competencia digital, y género. Ha participado en numerosos proyectos Internacionales y Nacionales, y colabora activamente con centros extranjeros de reconocido prestigio. 


\section{Isabel Cerezo Cortijo}

Universidad de Extremadura

Graduada en Educación Infantil por la mención de Habilidades Comunicativas y Lenguas Extranjeras por la Universidad de Extremadura (2019). Máster Universitario en Investigación en Ciencias Sociales en la especialidad de Psicología por la Universidad de Extremadura (2020). En la actualidad se encuentra en fase de formación investigadora en el ámbito de la tecnología educativa.

\footnotetext{
(c) (1) (9)

Los textos publicados en esta revista están sujetos a una licencia de Reconocimiento 4.0 España de Creative Commons. Puede copiarlos, distribuirlos, comunicarlos públicamente y hacer obras derivadas siempre que reconozca los créditos de las obras (autoría, nombre de la revista, institución editora) de la manera especificada por los autores o por la revista. La licencia completa se puede consultar en:Licencia Creative Commons Atribución-NoComercial-Compartir por igual 4.0 Internacional.
} 